\title{
Evaluación y gestión de la vulnerabilidad de los distritos 5 y 6 del municipio de El Alto
}

Évaluation et gestion de la vulnérabilité des districts 5 et 6 de la municipalité d'El Alto

Appraisal and management of the vulnerability of districts 5 and 6, El Alto, Bolivia

\section{Sébastien Hardy}

\section{(2) OpenEdition \\ Journals}

\section{Edición electrónica}

URL: http://journals.openedition.org/bifea/2538

DOI: 10.4000/bifea.2538

ISSN: 2076-5827

Editor

Institut Français d'Études Andines

Edición impresa

Fecha de publicación: 1 diciembre 2009

Paginación: 873-892

ISSN: 0303-7495

Referencia electrónica

Sébastien Hardy, «Evaluación y gestión de la vulnerabilidad de los distritos 5 y 6 del municipio de El Alto », Bulletin de l'Institut français d'études andines [En línea], 38 (3) | 2009, Publicado el 01 junio 2010, consultado el 17 noviembre 2020. URL : http://journals.openedition.org/bifea/2538 ; DOI : https:// doi.org/10.4000/bifea.2538

\section{BY NO ND}

Les contenus du Bulletin de l'Institut français d'études andines sont mis à disposition selon les termes de la licence Creative Commons Attribution - Pas d'Utilisation Commerciale - Pas de Modification 4.0 International. 


\title{
Evaluación y gestión de la vulnerabilidad de los distritos 5 y 6 del municipio de El Alto
}

\author{
Sébastien Hardy*
}

\section{Resumen}

Representativa de la pobreza urbana de las ciudades periféricas, la ciudad de El Alto se convirtió en uno de los lugares más emblemáticos de las reivindicaciones sociales en Bolivia. Sin embargo, estos aspectos a menudo hacen olvidar que, con su fuerte crecimiento demográfico espacial, la ciudad se expone cada vez más a distintas amenazas de origen natural y antrópico.

En el marco de un proyecto Dipecho del Departamento de Ayuda Humanitaria de la Comisión Europea, el equipo del programa Pacivur de la unidad de investigación UR029-IRD se encargó de identificar las zonas de riesgo de dos de los once distritos que componen El Alto. La definición de estas zonas de riesgos proporcionó los elementos para aplicar una mejor preparación ante los posibles desastres. Por lo tanto, se requirió conocer al mismo tiempo los procesos físicos originados por las amenazas, los elementos que éstos pueden dañar y que causan el desastre, así como los elementos útiles para la gestión de la posible crisis que deriva de éstos.

Palabras clave: amenaza, daño, vulnerabilidad, ciudad, Bolivia

\section{Évaluation et gestion de la vulnérabilité des districts 5 et 6 de la municipalité $d^{\prime}$ El Alto}

\section{Résumé}

Représentative de la pauvreté urbaine des villes périphériques, la ville d'El Alto s'est convertie en I'un des lieux les plus emblématiques des revendications sociales en Bolivie. Ces aspects font pourtant souvent oublier qu'avec sa forte croissance démographique et spatiale, la ville s'expose toujours plus à divers aléas d'origine naturelle ou anthropique.

* Institut de Recherche pour le Développement (IRD), UR 029, programa Pacivur, Av. Hernando Siles 5290 esq. calle 7, CP 9214 Obrajes, La Paz, Bolivia. E-mail: sebastien.hardy@ird.fr 
Dans le cadre d'un projet Dipecho du Département d'aide humanitaire de la Commission européenne, l'équipe du programme Pacivur de I'UR029-IRD a été chargée d'identifier les zones à risques de deux des onze districts qui composent El Alto. L'identification de ces zones à risques a fourni les éléments pour mettre en œuvre une meilleure préparation face aux désastres possibles. Elle a donc nécessité de connaître à la fois les processus physiques à l'origine des aléas, les éléments que ces derniers peuvent endommager et qui provoquent le désastre, ainsi que les éléments utiles à la gestion de la situation d'urgence et/ou de crise éventuelle qui en découle.

Mots clés : aléa, dommage, vulnérabilité, ville, Bolivie

\title{
Appraisal and management of the vulnerability of districts 5 and 6, EI Alto, Bolivia
}

\begin{abstract}
Representative of urban poverty in the suburbs of La Paz, El Alto has become one of the most emblematic places of social demands in Bolivia. However, these aspects often result in forgetting that with its high demographic and spatial growth, the city is more and more exposed to various threats of natural or anthropic origin.

Within the framework of a DIPECHO project from the Department of Humanitarian Aid of the European Commission, the team of the PACIVUR program from the UR029-IRD was put in charge of identifying risk zones in two of the eleven districts which make up El Alto. The identification of risk zones has provided elements to implement a better preparedness against possible disasters. Thus, it required knowledge of physical processes from the outset of threats and the elements that can be damaged and which can cause disasters, as well as useful elements for the management of possible resultant crises.
\end{abstract}

Key words: hazard, injury, vulnerability, city, Bolivia

\section{INTRODUCCIÓN}

La temática de los riesgos de origen natural y de origen antrópico1 parece más pertinente cada día. El listado de los daños que generan aumenta año tras año (Blaikie et al., 1994; Dubois-Maury \& Chaline, 2002; Pelling, 2003; Pigeon, 2007) y cuestionan los modelos de desarrollo a través del mundo (Davis, 2006a). Esta tendencia es bastante lógica ya que sigue el aumento de la población mundial: tanto más habitantes hay, mayor es la cantidad de elementos del funcionamiento de las sociedades que están expuestos a los daños (Dauphiné, 2001; D’Ercole

1 En este texto, las expresiones riesgo(s)/amenaza(s)/evento(s) de origen natural y antrópico son utilizadas por comodidad debido a que no se dispone de expresiones más precisas. Por ejemplo, en los procesos que producen la amenaza, se reconoce que es difícil tener en cuenta lo que corresponde a la dinámica terrestre y lo que corresponde a las actividades humanas. La extensión de la población humana en la superficie de la tierra, y en particular de las actividades humanas, induce a pensar que los procesos son ampliamente forzados por aquella. No obstante, haciendo variar las escalas de análisis, se constata que hay procesos en los cuales la influencia humana es menos clara. Toda una rama de la investigación sobre los riesgos se dedica a discutir la terminología y sus implicaciones metodológicas. Remitimos al lector a estos trabajos, particularmente los de Patrick Pigeon (2005). 
\& Metzger, 2004). Como la población se va concentrando cada vez más en las ciudades (Davis, 2006b; Pigeon, 2007), estas sufren daños mayores con una frecuencia acelerada por acumulación a largo plazo.

Frente a esta situación, la respuesta más usual se ha construido a partir de una definición del riesgo establecida alrededor de la dupla amenaza-vulnerabilidad. En realidad se ha intervenido con mayor frecuencia sobre las amenazas (Pigeon, 2005; Hardy, por publicarse). Por esta razón, gran parte de los trabajos de investigación científica se dedica a establecer un mejor conocimiento de la amenaza, aunque este enfoque sea muy insuficiente. Cualquiera que sea el país considerado en el mundo, este enfoque ha conducido a la construcción de obras de protección destinadas a resistir a las amenazas (Hardy \& Musset, 2008). Ahora bien, las obras de protección, por útiles que fueran en ciertos casos, han mostrado límites considerables (Dauphiné \& Provitolo, 2007). Incluso han levantado algunos interrogantes respecto a efectos inéditos sobre el aumento de los daños (Pigeon, 2005). Y sin embargo, los gestores de los riesgos han continuado privilegiando este enfoque.

En el marco de dos programas2 del Instituto de Investigación para el Desarrollo (IRD) en los países andinos, un equipo de la UR 029 Medio ambiente urbano dirige un trabajo de reflexión sobre el riesgo que pretende superar las contradicciones causadas por la definición clásica (D'Ercole \& Metzger, 2009). Esta reflexión conceptual se traduce por la aplicación de nuevas metodologías de análisis del riesgo ( $D^{\prime}$ Ercole et al., 2009) en el cual el riesgo ya no es definido de manera clásica sino como la posibilidad de perder aquello que se considera importante (D'Ercole \& Metzger, 2004). Este nuevo enfoque conceptual ha permitido no concentrarse con exclusividad sobre en la amenaza y enfocarse sobre el funcionamiento de la sociedad. También ha orientado los resultados hacia una planificación preventiva más eficaz respondiendo mejor a las necesidades de los gestores y de las poblaciones. Algunos trabajos de investigación realizados para un proyecto Dipecho3 en la ciudad boliviana de El Alto, han procurado verificar y profundizar la aplicabilidad de este enfoque conceptual.

Los proyectos Dipecho son financiados por el Departamento Humanitario de la Comisión Europea $(\mathrm{ECHO})$ con el objetivo de preparar mejor a las poblaciones de los países del Sur para hacer frente a las catástrofes. El equipo IRD-Pacivur respondió, en octubre de 2007, a una licitación de ECHO para identificar las zonas de riesgo en los distritos 5 y 6 de El Alto. Desarrolló una metodología específica para alcanzar los objetivos planteados por este proyecto. El equipo no solamente aspiraba a reconocer las amenazas de origen natural y antrópico en los distritos 5 y 6 , sino también a identificar las poblaciones que se exponían a estas amenazas, así como a establecer los medios disponibles para hacer frente a una

2 Entre 1999 y 2004, el programa Sistema de información geográfico y riesgos en el Distrito Metropolitano de Quito y desde 2006, el programa Pacivur (programa andino de capacitación y de investigación sobre la vulnerabilidad y los riesgos en medio urbano) en La Paz, Lima y Quito.

3 Dipecho: Disaster Preparedness Program of the European Commission's humanitarian aid department. 
posible crisis provocada por la ocurrencia inesperada de un evento perjudicial, a fin de prepararse mejor para hacer frente a la catástrofe.

Esta operación de investigación aplicada resultó en productos destinados a los beneficiarios del proyecto Dipecho, a las autoridades municipales de El Alto y a la Defensa civil boliviana: bases de datos administradas con ArcGis 9.2, una serie de mapas con reseñas, capacitación en la metodología y en el uso de datos. Se constituyó un equipo pluridisciplinario, guiado por investigadores de las ciencias sociales asociados a investigadores de las ciencias de la tierra, a fin de crear la información necesaria para este proyecto. Su aspecto innovador residió sobre todo en la reflexión metodológica que ha orientado los trabajos: los habitantes de los distritos 5 y 6 de El Alto definieron el riesgo como la posibilidad de perder lo que les parece importante para el funcionamiento de este espacio urbano, es decir lo que tiene carácter esencial (D’Ercole \& Metzger, 2004).

A partir de talleres durante los cuales los investigadores demostraron a las poblaciones que los elementos esenciales del territorio son aquellos cuya pérdida les es perjudicial, las poblaciones han definido una gran variedad de ellos, en primer lugar ellas mismas, por el hecho de estar expuestas a las amenazas. En esta ocasión se diferenció la población residente de la población flotante. Por ejemplo, el distrito 6 de El Alto tiene la particularidad de acoger dos días por semana al mayor mercado de Bolivia: la Feria 16 de Julio. Durante esos dos días el distrito 6 recibe poblaciones no residentes, es decir flotantes. Otro ejemplo de población flotante: los niños de los dos distritos seleccionados van a la escuela los días de semana mientras que sus padres van a su lugar de trabajo, etc. Por otro lado, otros elementos también fueron definidos como esenciales.

Se dedujo de esta primera fase de trabajo que había que tomar en cuenta la variación espacial de los elementos esenciales en función de la temporalidad. Por ejemplo, nos ha parecido importante tratar de pensar en la vulnerabilidad del elemento esencial que presenta la población y esto en función de la temporalidad. Efectivamente, la vulnerabilidad de un elemento puede difundirse a otros a causa de su temporalidad de funcionamiento. Por lo tanto, esta cuestión de temporalidad es particularmente importante para abordar la vulnerabilidad (Lagadec, 2002: 164). Sin embargo, la temporalidad es raramente abordada, cuando en realidad en situación de urgencia y/o de crisis juega un papel importante. Es este vínculo entre la vulnerabilidad y la temporalidad el que nos guiará en la demostración a lo largo de este texto.

Después de haber presentado las dinámicas demográficas y espaciales de El Alto, para mostrar cómo las condiciones de la vulnerabilidad se construyen a partir del contexto de la pobreza, abordaremos más directamente la problemática de los riesgos identificando algunos elementos esenciales y cómo son susceptibles de perderse. Por eso se establecerán más específicamente los vínculos entre la vulnerabilidad de los elementos esenciales y la temporalidad, en un modo que permita interesarse en las condiciones de la vulnerabilidad susceptibles de fragilizar los elementos expuestos. Se hará con el objetivo de orientar la gestión de los riesgos hacia la preparación para las situaciones de urgencia y/o de crisis. 


\section{EL ALTO: PERIFERIA URBANA EN UN PAÍS DEL SUR}

\section{1. Crecimiento de la población y dinámica espacial}

Las diferentes crisis que han sufrido las regiones del Altiplano boliviano —reforma agraria de 1953, caída de la actividad minera durante el decenio de 1980 (Franqueville, 2000; Arbona \& Kohl, 2004)— han llevado a sus habitantes a migrar por olas sucesivas hacia el municipio de La Paz y, debido a la ocupación plena del espacio urbano, de manera centrífuga, a instalarse en sus alrededores (Sandoval \& Sostres, 1989; Sandoval \& Ayllon, 1992). Por lo tanto, el crecimiento de El Alto se traduce primero en un esparcimiento demográfico operado desde La Paz, por la llegada brusca de olas migratorias y, finalmente, más recientemente, por el crecimiento natural de una población con una estructura que se mantuvo muy joven. Este crecimiento demográfico sigue siendo bastante sostenido, con una tasa de crecimiento establecida entre los dos censos de 1992 y 2001 en 5,1\%, tasa que hay que comparar con el promedio departamental que alcanza el 2,3\% en el mismo periodo (INE, 2001; Garfías \& Mazurek, 2005). En menos de 60 años, El Alto ha multiplicado así su población por más de 80 , haciéndola pasar de aproximadamente 11000 habitantes en 1950 a más de 896773 habitantes estimados en 2008, superando el número de habitantes del municipio histórico de La Paz (INE, 2008) (fig. 1).

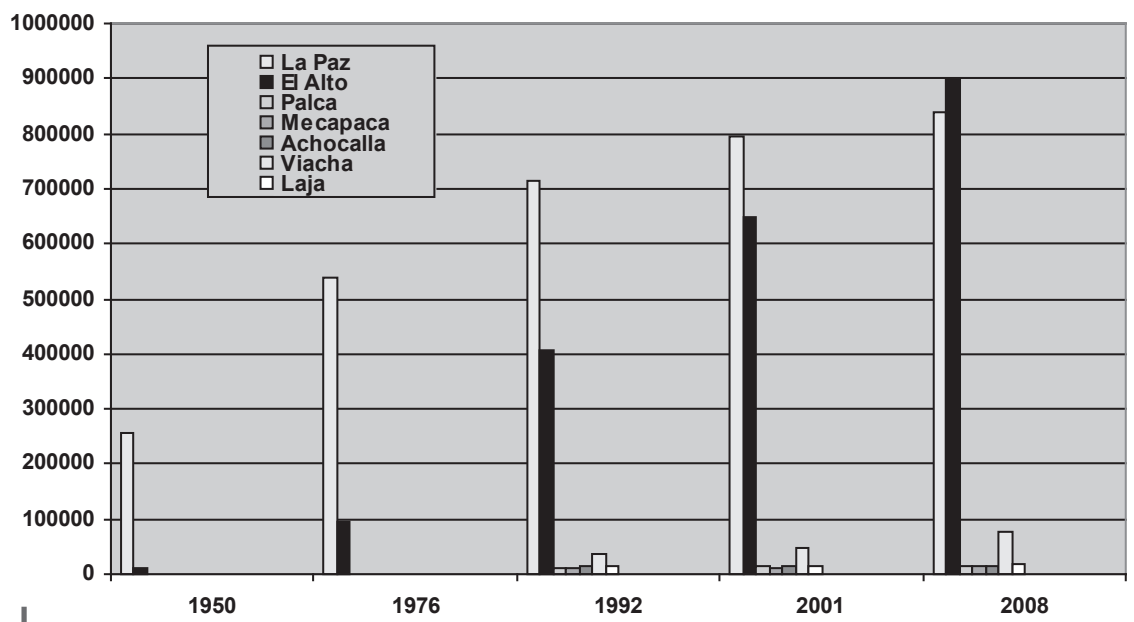

Figura 1 - Evolución de la población de los municipios de la aglomeración de La Paz 4

Fuente: INE, diferentes datos de censos, URL: http://www.ine.gov.bo

Concepción: S. Hardy, IRD, 2009

4 La evolución demográfica es deformada por la evolución de las áreas de recolección de información estadística ya que El Alto no se convirtió en municipalidad sino en 1988. 
Este crecimiento demográfico trajo consigo un crecimiento espacial sostenido. En apenas 30 años, la superficie urbana ha sido multiplicada por 5,2 pasando de 2700 hectáreas en 1976 a 14130 hectáreas en 2007.

\section{2. Deficiencia de la planificación urbana e intervencionismo de los habitantes}

Durante este lapso de tiempo, la planificación urbana ha sido muy insuficiente (Durán et al., 2007). Varias razones lo explican.

La Paz y El Alto han constituido un mismo municipio hasta 1988. Hasta esta fecha, la herencia hispánica de segregación de las poblaciones (Mayorga, 1997) explicaba que los barrios principalmente habitados por poblaciones de origen indígena (en su mayoría aymara, luego quechua) no habían sido objeto de una planificación rigurosa. Por otro lado, mientras las políticas de vivienda eran casi inexistentes (Durán et al., 2007), por oportunismo político en un periodo de gran inestabilidad municipal, los alcaldes sucesivos (Mayorga, 1997: 287) no intervinieron para impedir la urbanización ilegal de las laderas abruptas (del actual municipio de La Paz), y de los lechos mayores de los torrentes (a la vez en los municipios actuales de La Paz y de El Alto) (GMLP, 2007; Hardy, 2008; Hardy, 2009a), no obstante considerados como inconstructibles (BCEOM, BRGM, PCA, 1977). Las poblaciones en búsqueda de una vivienda pudieron instalarse en zonas expuestas a amenazas de origen natural y antrópico.

Por lo demás, acostumbrados a velarse por sí mismos, los habitantes siempre aceptaron de mal agrado las decisiones de urbanismo o de equipamiento impuestas por las instancias municipales (Poupeau, 2008a). Es por esta razón que El Alto se ha construido extensamente gracias a las tenaces luchas de habitantes pobres —el 65 \% de la población se define como pobre (INE, 2001)— estructurados en asociaciones de habitantes (juntas vecinales). Las que han permitido la urbanización de El Alto y le han dado su apariencia inacabada y desordenada (Arbona \& Kohl, 2004; Durán et al., 2007) son las movilizaciones de habitantes que reivindicaban la satisfacción de sus necesidades y se zafaron obstinadamente de las autoridades representativas legales (García Linera, 2006; Poupeau, 2007; Poupeau, 2008b). En efecto, por lo general, los propios habitantes de El Alto han construido sus viviendas en terrenos cuya propiedad todavía no había sido legalizada (Durán et al., 2007). Por otra parte, todavía existe un mercado de bienes raíces particularmente dinámico en manos de loteadores que venden ilegalmente terrenos a los recién llegados, obligando a menudo a los servicios municipales a levantar un acta a posteriori de la urbanización. Los loteadores no se preocupan de las consecuencias a mediano y largo plazo, por ejemplo al lotear un terreno en un lecho importante de río, ni del uso que puede darse al terreno (actividades industriales peligrosas yuxtapuestas a viviendas, etc.). En una segunda etapa, los habitantes de un barrio naciente, estructurados en asociaciones de habitantes por lo menos desde el decenio 1950, se han concentrado para exigir la instalación de los servicios urbanos, como la electricidad y el agua. También han realizado 
trabajos colectivos con el fin de equipar su barrio, generalmente los fines de semana, con autofinanciamiento: empedrado de las calles, construcción de una casa vecinal, aceras, realización de una plaza de barrio con juegos para los niños y campos deportivos, etc. Por lo tanto, los servicios municipales se encontraron confrontados a la obligación de levantar posteriormente el acta jurídica de este urbanismo alteño, a pesar de que las pocas normas establecidas no hubieran sido respetadas.

\section{3. Un municipio nuevo y pobre}

La creación del municipio autónomo de El Alto en 1988 (Baby, 1995) generó nuevas dificultades en el control del crecimiento urbano. En un primer tiempo, hubo que organizar nuevos servicios municipales: planificación, catastro, urbanismo, etc., que hasta entonces eran servicios operados por la municipalidad de La Paz. Sin embargo, la nueva municipalidad de El Alto es pobre en recursos (Obermaier, 1999: 63), más aún cuando la recaudación de impuestos es difícil, como lo prueban las protestas del 8 de septiembre de 2003 contra el aumento del régimen tributario local (García Linera, 2006). Por lo tanto, la municipalidad no ha logrado estructurar un equipo suficientemente experimentado para responder a la situación, a pesar de que el fuerte crecimiento demográfico espacial exigía actuar rápida y eficazmente a la vez. La inexperiencia de los técnicos novatos de la municipalidad alteña explica en parte las incoherencias actuales de la urbanización.

No obstante, las autoridades encontraron soluciones a fin de mejorar el urbanismo. Por ejemplo, la Fundación católica Cuerpo del Cristo fue designada por la Presidencia de la República a través de la Comisión boliviana encargada de combatir la pobreza, para planificar el desarrollo de la ciudad de El Alto. Aquella produjo diversos documentos, como el Plan de regulación en junio de 1999 y un Plan de ordenamiento urbano en junio del 2000. Estos análisis han permitido reflexionar sobre el uso del suelo, partiendo de la comprobación de su descontrol: división de las tierras que desde el principio no respetaban las formas y las dimensiones adecuadas; construcción de los barrios que no tenían en cuenta ni la topografía ni las actividades; equipamientos de barrios realizados por los habitantes, desarticulados del resto de la trama urbana; estructura de los caminos insuficiente y sin continuidad; bajas densidades que hacían difícil un ordenamiento eficaz (Obermaier, 1999: 36). Las propuestas de ordenamiento del territorio alteño, fundadas en una mejor repartición de las poblaciones y de sus actividades, a través principalmente de una nueva repartición de los distritos (Obermaier, 1999: 64-68), a pesar de las necesidades constatadas, nunca fueron plenamente aceptadas por las poblaciones, y por lo tanto no fueron aplicadas. Es por eso que se siguen construyendo viviendas cerca de las fábricas, en el lecho de los ríos, sobre antiguos vertederos, lo que implica en parte el aumento de la frecuencia más elevada de eventos al origen de daños (Hardy, 2008). 


\section{LA PROBLEMÁTICA DE LOS RIESGOS EN EL ALTO}

\section{1. Los eventos pasados}

La ciudad de El Alto se expande en el Altiplano boliviano, a más de 4000 metros de altitud. Aunque situada en la zona intertropical, a $16^{\circ}$ de latitud Sur, la altitud y la proximidad del litoral pacífico con sus aguas frías crean las condiciones de una semiaridez: las lluvias nunca son muy voluminosas (promedio anual inferior a $500 \mathrm{~mm}$ ) pero sí pueden ser muy violentas (Olmos, 2008). Frío, sol, viento y violentas precipitaciones (Montes de Oca, 2005) hacen muy difícil la vida en El Alto.

En este contexto, el fuerte crecimiento demográfico y espacial en un territorio urbano poco controlado explica que los eventos que generan daños sean frecuentes5. El observatorio sísmico San Calixto y la Defensa civil boliviana realizaron una compilación de los eventos catastróficos en Bolivia entre el $1^{\text {ro }}$ de enero de 1970 y el 31 de diciembre de 2007, principalmente a partir del periódico El Diario. Estos datos figuran en la base de información DesInventar. Por otra parte, un trabajo de investigación efectuado en El Alto, en los distritos 5 y 6 , a partir de los archivos de los bomberos y del periódico El Alteño, complementó estos datos (Hardy, 2008). Su análisis revela que El Alto es regularmente golpeado por eventos de origen natural y antrópico (fig. 2).

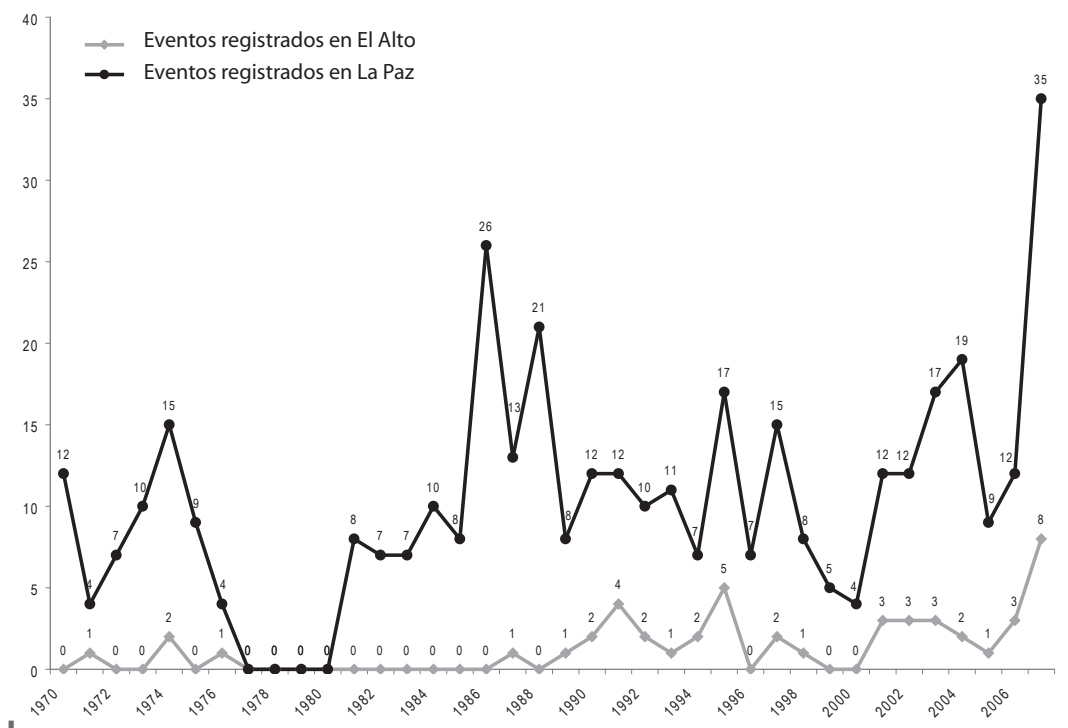

Figura 2 - Eventos que han afectado a las ciudades de La Paz y El Alto 1970-20076

Fuentes: Base de datos modificada DesInventar, <URL: http://www.desinventar.org> Concepción: S. Hardy, IRD, 2009

5 Ver en este volumen la introducción general de R. D’Ercole, S. Hardy y J. Robert, pp. 401-410.

6 Las poblaciones de El Alto, una ciudad autoconstruida, probablemente, declaran menos deterioros que las un poco más acomodadas de La Paz. 
Si los eventos que afectaron El Alto son menos numerosos que los registrados en La Paz, es a la vez porque las condiciones físicas en El Alto son ligeramente más favorables que en La Paz (Hardy, 2009a) (topografía casi plana y erosión regresiva menos pronunciada), pero también porque su urbanización más reciente favorece menos, por el momento, la exposición de elementos esenciales a las amenazas de origen natural y antrópico. Sin embargo el abanico de las amenazas que han causado daños en el pasado es bastante amplio (fig. 3).
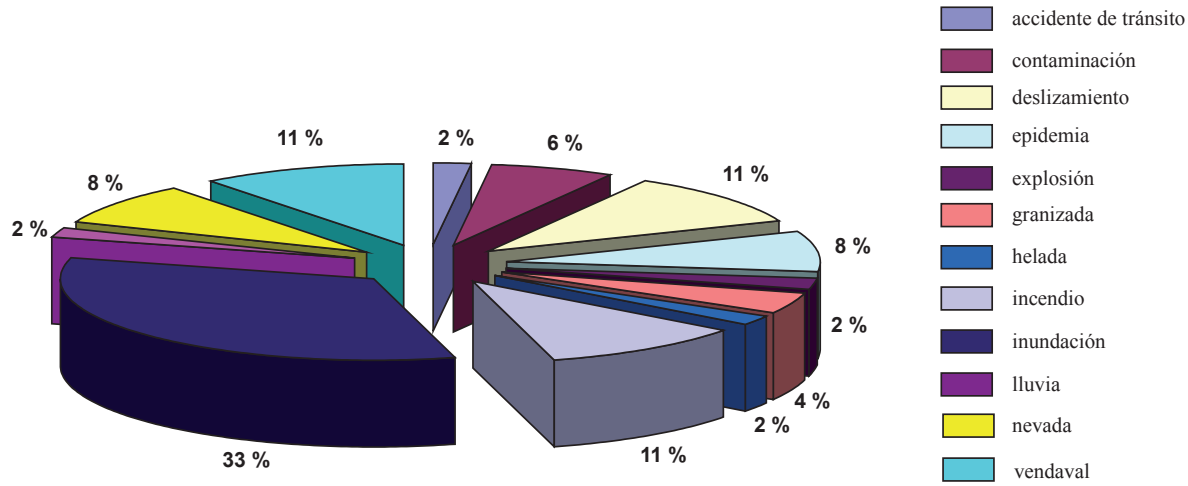

Figura 3 - Repartición por tipo de amenaza de los eventos que han afectado a la ciudad de El Alto 1970-2007

Fuentes: Base de datos (modificada) DesInventar, <URL: http://www.desinventar.org >

Concepción: S. Hardy, IRD, 2009

Un rápido panorama muestra que los daños se debieron esencialmente a las bajas temperaturas (2002), a las tormentas de viento (1991, 1994, 1995, 2007), a las precipitaciones de nieve (1990, 1991, 1993, 2007), de lluvia (1987), de granizo (1971, 2007), a las inundaciones (1983, 1992, 1995, 1997, 2001, 2003, 2004, $2005,2006)$ y a los deslizamientos de tierra $(1974,1976,1997,2002,2003,2006)$ consecutivos. Algunos eventos son claramente de origen antrópico (incendios y explosiones).

A veces estos eventos han llevado los habitantes y las autoridades gestoras a situaciones de urgencia y/o de crisis. Durante su gestión 2006-2007, la unidad de bomberos de El Alto ha registrado 134 urgencias relacionadas a inundaciones, la mayoría de ellas en los distritos 5 y 6 , al norte del espacio urbano del municipio de El Alto (fig. 4) (Mollericona, 2008).

Los distritos 5 y 6 , por su topografía, por las poblaciones y por los elementos del funcionamiento urbano que concentran, llamaron particularmente la atención.

\section{2. La preocupación por los riesgos}

En la municipalidad de El Alto, la preocupación por los riesgos tuvo simultáneamente como respuesta la canalización de los principales torrentes alrededor de los cuales 


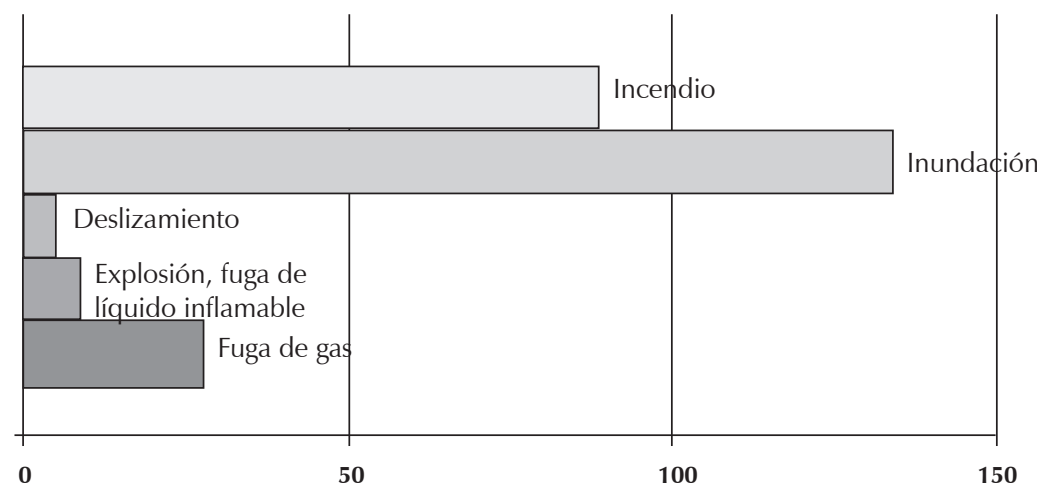

Figura 4 - Las situaciones de urgencia por tipo que han necesitado la intervención de los bomberos, El Alto 2006-2007

el tejido urbano se ha edificado, así como la estabilización de las laderas por medio de gaviones. No obstante, estas medidas de gestión de los riesgos nunca han dado resultados verdaderamente satisfactorios como lo muestra el aumento de los eventos que causan daños.

Esta situación no es para nada paradójica. Por ejemplo, en el caso de las obras de ingeniería destinadas a resistir las inundaciones, dado que solo hay una estación hidrometeorológica en El Alto7, su calibración se efectuó a partir de datos cuantitativa y cualitativamente insuficientes. Por otro lado, recientes análisis de datos climáticos concluyeron en una tendencia al aumento de la intensidad de las precipitaciones y en su concentración en el tiempo (Olmos, 2008). Reuniendo estas dos explicaciones, se deduce un aumento ${ }^{8}$ de los daños, a pesar de las medidas de gestión de los riesgos.

En estas condiciones, el trabajo de investigación llevado a cabo en el marco del proyecto Dipecho en los distritos 5 y 6 se ha dedicado a identificar mejor no solamente las amenazas tanto de origen natural como antrópico susceptibles de causar daños (Pigeon, 2005), sino también los elementos que se exponen a las amenazas y sus condiciones de vulnerabilidad que explican la mayor fragilidad de ciertos de ellos. Esta identificación de los principales elementos vulnerables - principalmente los que son necesarios para la gestión de una situación de urgencia y/o de crisis, y que la población no puede perder por el riesgo de alterar fuertemente su capacidad de manejar la urgencia, luego el retorno a una situación normal (la recuperación) (D’Ercole \& Metzger, 2004) — ha permitido llegar a recomendaciones en términos de planificación preventiva (Hardy, 2008).

7 La estación meteorológica situada en el aeropuerto de El Alto es administrada por el Servicio Nacional de Meteorología e Hidrología (Senamhi).

8 En valor absoluto, el aumento de los daños traduce un aumento de elementos expuestos y de su valor. En cambio, el crecimiento demográfico hace disminuir el valor de los daños por habitantes. 


\section{3. Los elementos expuestos en los distritos 5 y 6 de El Alto}

Los distritos 5 y 6 , situados al norte de la parte urbana de la municipalidad de El Alto, concentran entre ambos, en 2001, 190263 habitantes, es decir cerca del $30 \%$ de la población alteña reunida en un $16 \%$ de su superficie (INE, 2001). La población se reparte según dos direcciones: la densidad es decreciente de sur a norte, desde el aeropuerto; también es decreciente de este a oeste (fig. 5). Esta configuración tiene una clara explicación en la historia de la urbanización, centrífuga desde La Paz y desde los grandes ejes de comunicación que conectan el Altiplano con La Paz, atravesando El Alto. Esta población residente constituye ya un primer elemento esencial, el más importante.

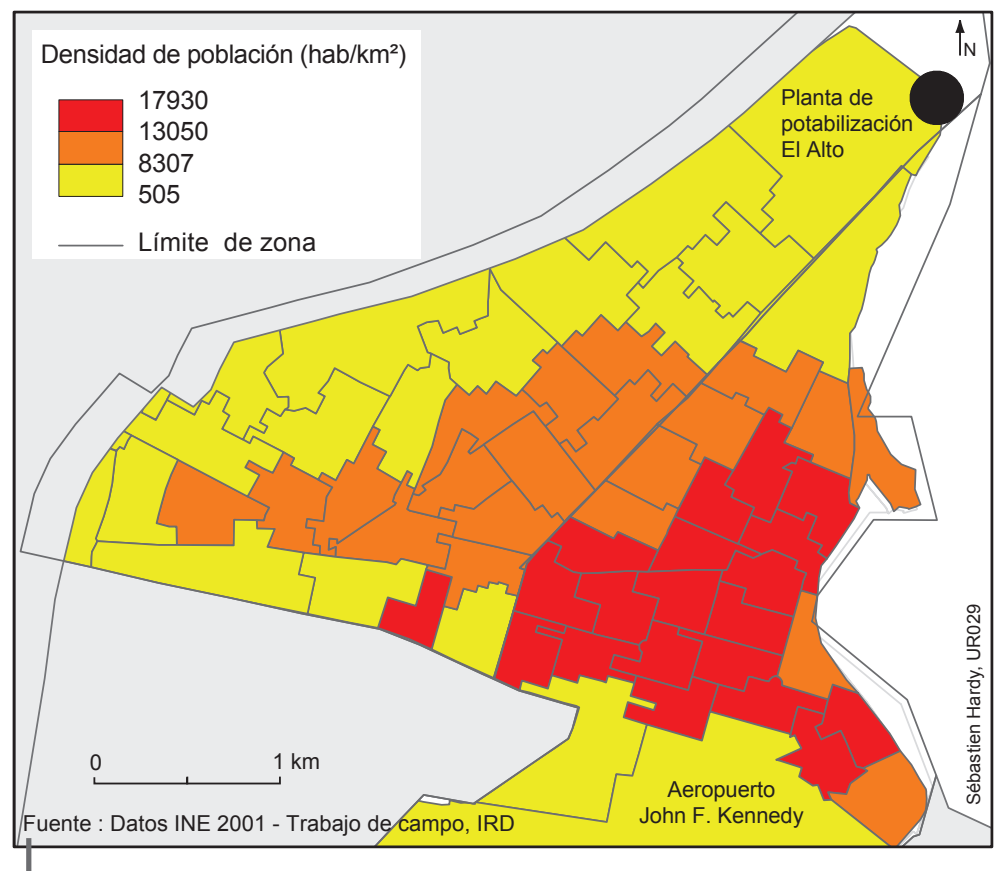

Figura 5 - Las densidades en los distritos 5 y 6 de El Alto

Además de esta población de residentes, estos dos distritos concentran puntualmente en el tiempo poblaciones calificadas de flotantes, que pueden ser importantes en número. Por ejemplo, el distrito 6 es la sede de uno de los más importantes mercados del país, la Feria 16 de Julio, que, dos veces por semana concentran una multitud de comerciantes y de compradores. El distrito 5, por su parte, reúne numerosas fábricas que emplean a grandes cantidades de personas que provienen de toda la aglomeración de La Paz, como la planta de fabricación de Coca-Cola, también la de Pepsi-Cola y la de productos lácteos Pil. La población flotante constituye un elemento expuesto, así como las actividades económicas presentes en la zona de estudio. 
En caso de ocurrencia de un evento asociado a daños, estos elementos, por su exposición a la amenaza deben ser tomados en cuenta por los gestores de los riesgos. Efectivamente, estas informaciones son

«particularmente interesantes para la gestión de crisis y la orientación de las operaciones de auxilio, abordando un punto a menudo mal conocido y poniendo en evidencia nuevas vulnerabilidades» (Robert, 2007: 44).

Estas pueden resultar en el despliegue de medios de auxilio más adecuados: fugas eventuales de productos peligrosos, heridas específicas que requieren tratamiento, evacuación de poblaciones que no residen en la zona del accidente, etc.

En definitiva, estos dos distritos concentran cada uno equipamientos útiles para su propio funcionamiento cotidiano (mercados, infraestructuras urbanas, etc.), y también útiles, en menor escala, para el resto de la aglomeración. Se trata del aeropuerto internacional El Alto y de la planta El Alto de potabilización del agua.

La planta El Alto de potabilización del agua abastece de agua no solamente a una gran parte de El Alto, sino también a la parte occidental de La Paz. Por lo tanto, existe el riesgo de perder este elemento, a escala de los dos distritos estudiados de El Alto que son abastecidos de agua potable por esta planta, pero también a escala del municipio de El Alto y de una parte de La Paz (Hardy, 2009b). De la misma manera, la pérdida por exposición a la amenaza de la fábrica Pil, por ejemplo, puede provocar disfuncionamientos locales, sino también a nivel de la aglomeración interrumpiendo el principal aprovisionamiento de productos lácteos. Por lo tanto, se dibujan los elementos esenciales existentes en los distritos 5 y 6 de El Alto, en diferentes niveles espaciales y también temporales.

\section{4. La vulnerabilidad en El Alto: la exposición a numerosas amenazas}

Los elementos presentes en los distritos 5 y 6 pueden además estar expuestos a amenazas (Hardy, 2008). Estas amenazas han sido identificadas lo más precisamente posible —en las condiciones dadas del proyecto Dipecho- por estudios específicos de geología (Argollo, 2008) y de hidrología (Olmos, 2008). Una parte de la investigación se ha interesado también en las amenazas de origen antrópico (Hardy, 2008).

Las amenazas de origen natural reúnen dos fenómenos: la inundación y la inestabilidad del suelo. Estos dos distritos son atravesados de noreste a suroeste por torrentes de la cuenca de la Cordillera andina, susceptibles de desbordarse cuando los aportes son un poco más elevados de lo normal, a causa de precipitaciones un poco más voluminosas, así como la rapidez de concentración de las aguas de escorrentía. Su carga puede volverse importante. Sus depósitos a lo largo del Cuaternario explican que ciertos terrenos sean más inestables que otros.

Se considera que estas amenazas son de origen natural (Pigeon, 2005), pero los análisis demuestran que en El Alto, ciertos procesos son formados por el hombre. 
Por ejemplo, el estudio de geología-geomorfología ha identificado, además de los deslizamientos de tierra en parte ligados a la estructura de las rocas existentes, la inestabilidad de las tierras que favorece la ocupación de antiguos depósitos de basura no comprimidos y mal recubiertos (fig. 6). Por su parte, el estudio de hidrología ha permitido identificar los espacios susceptibles de inundarse, que en gran parte son el resultado de una mala calibración durante el encauzamiento de los torrentes que atraviesan la parte norte de El Alto. Estas amenazas constituyen, sin ninguna duda, una coerción para las actividades humanas, pero también son en parte el resultado de éstas.

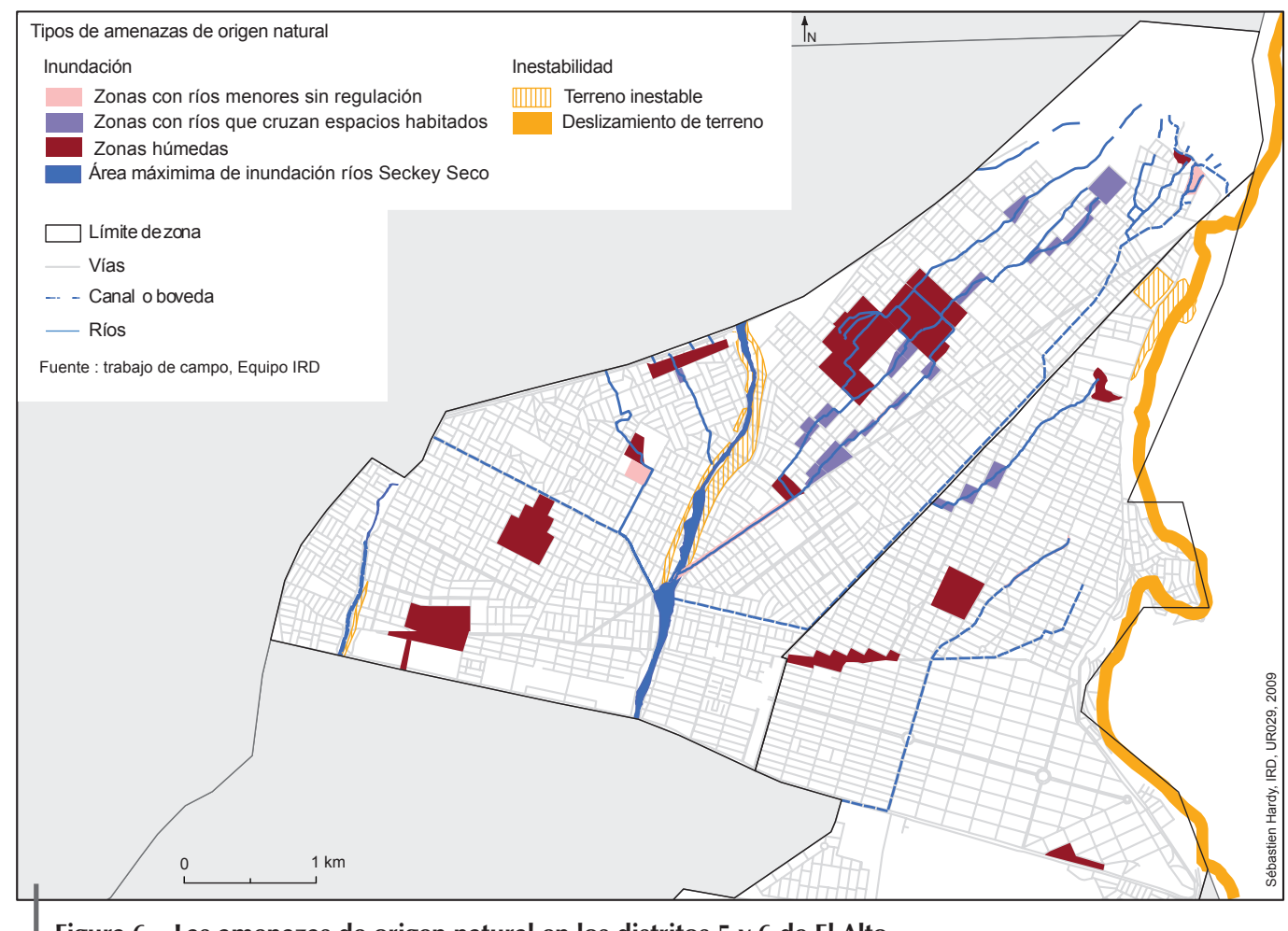

Figura 6 - Las amenazas de origen natural en los distritos 5 y 6 de El Alto

\section{VULNERABILIDAD Y TEMPORABILIDAD}

\section{1. Las temporalidades de los elementos expuestos}

Los espacios de riesgo no son forzosamente los espacios donde han sido identificadas las amenazas. Una amenaza puede tener incidencias, por transmisión, sobre otros elementos que aquellos que están inmediatamente presentes en la zona de exposición. Para comprender bien esta particularidad de los riesgos, conviene establecer cuál es la vulnerabilidad de los elementos (D'Ercole \& Metzger, 2004). 
Con la idea de ajustarse lo mejor posible a la preparación de la situación de urgencia y/o de crisis, el trabajo de investigación se ha interesado en diferentes condiciones que construyen la vulnerabilidad, en particular las relacionadas a la temporalidad. Tomar en cuenta las temporalidades permite en rigor una mejor comprensión de los espacios de riesgos (Lagadec, 2002: 164). Estos pueden estar disociados de los espacios considerados como bajo amenaza. Crear estas informaciones permite tanto a los organismos de auxilio como a las autoridades encargadas de la planificación, mejorar una eventual gestión de urgencia y/o de crisis y prepararse mejor para una catástrofe, a fin de limitar su impacto, tanto en fase de urgencia como en fase de recuperación.

La ocurrencia de una inundación en los distritos 5 y 6 de El Alto, de noche o de día, un lunes o un jueves, de ninguna manera producirá los mismos efectos. Esta evidencia ha orientado la construcción de bases de datos para desembocar en productos útiles a los beneficiarios. Así, tratándose de la población, la base ha diferenciado la población de noche — cuyos datos son proporcionados por el censo general de población realizado en 2001 (INE, 2001) — de la población de día. Para conocerla (características y ubicación), hubo que construir los datos y unirlos a otros, como los del funcionamiento de las actividades humanas en los distritos 5 y 6 de El Alto.

Por ejemplo, el mercado 16 de Julio que abre los jueves y domingos, hace que la circulación, en gran parte del distrito 6, sea prácticamente imposible. Esta actividad tiene repercusiones en ciertas características de los datos poblacionales. En efecto, un incendio en un centro educativo un día jueves, un parto problemático en un centro de salud un domingo, obligan a tomar medidas para auxiliar a las poblaciones expuestas: tanto las poblaciones residentes como las poblaciones flotantes. Ahora bien, la feria obstaculiza considerablemente el acceso de los auxilios. El mercado vuelve vulnerables muchos elementos presentes en su espacio (fig. 7). Localizando espacialmente los elementos susceptibles de perderse en la zona, caracterizándolos, identificando los factores que pueden volverlos vulnerables, se obtiene una mejor comprensión de los espacios de riesgo en función de las temporalidades — en este ejemplo un día de mercado/un día sin mercado_- Por lo tanto, la temporalidad hace variar la vulnerabilidad de los elementos presentes dentro de un espacio dado.

Este conocimiento de los espacios de riesgo y de su funcionamiento puede ser explotado entonces por los diferentes actores: establecer escenarios de crisis, identificar caminos de evacuación/de accesibilidad a los elementos9, negociar con los comerciantes caminos de acceso a estos elementos, preparar mejor a las poblaciones expuestas a tomar medidas en una crisis realizando simulaciones. De la suerte, se logra intervenir en ciertos factores de la vulnerabilidad de los elementos, para anticipar mejor las consecuencias.

9 Ver en este volumen el artículo de J.Nuñez y F. Demoraes, pp. 827-848. 


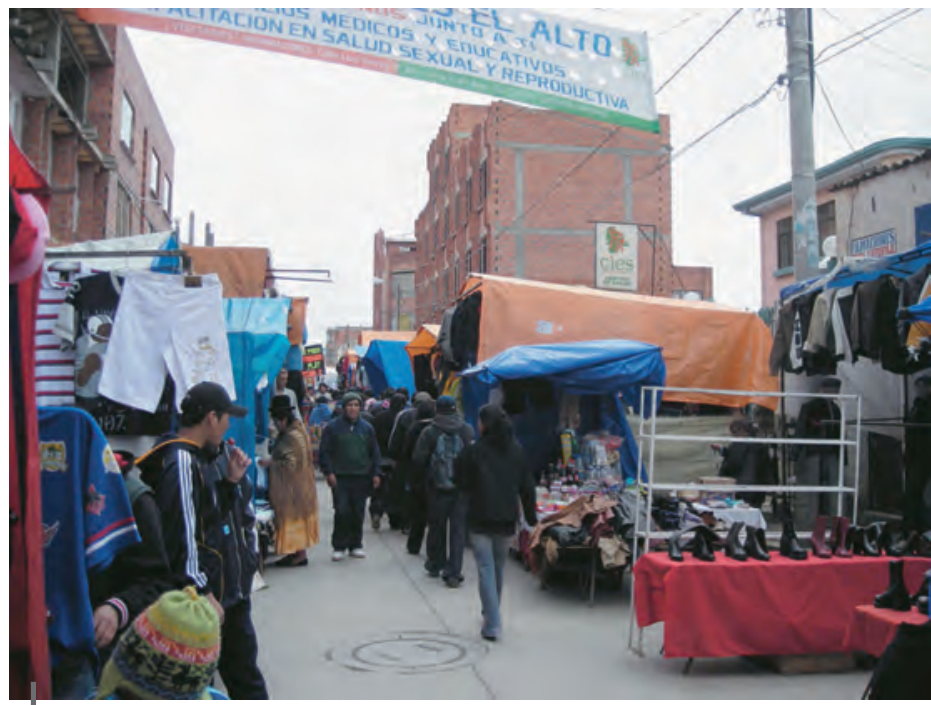

Figura 7 - El centro de salud CIES, situado en el corazón del distrito 6 de El Alto, es de difícil acceso para los organismos de auxilio y/o para las poblaciones en caso de urgencia y/o de crisis durante los días de la semana en que tiene lugar el mercado alteño 16 de Julio

Foto: S. Hardy, 2008

\section{2. La identificación de las temporalidades: una ayuda en la gestión de los riesgos}

Para ayudar a las poblaciones más expuestas a las amenazas, primero hay que identificarlas y comprender sus características en sus múltiples dimensiones, para enseguida prever soluciones para los problemas planteados. Una de las poblaciones menos preparadas para soportar las consecuencias de una crisis son los niños. Por lo tanto, hay que ayuydarles a prepararse para enfrentarlas. Por tal motivo, hay que saber principalmente dónde se encuentran los niños durante el día. Muchos de ellos frecuentan las escuelas que deben ser localizadas y que deben informar del número de niños inscritos.

Pero las escuelas en Bolivia funcionan por turnos: el de la mañana, el de la tarde y el de la noche. Construir una serie de mapas de las poblaciones existentes en las escuelas en función de los turnos da a los organismos encargados de los auxilios una mejor idea de los medios que deben desplegar dentro de una zona señalada por la ocurrencia de un evento.

Por ejemplo, la unidad educativa Portugal e Illimani, situada dentro de la zona urbana Nueva Asunción en el distrito 5 funciona solamente en la mañana (fig. 8). Se encuentra en un espacio expuesto a una inundación. Conociendo estos parámetros, en caso de una alerta en esta zona una tarde, los organismos de auxilio no necesitan enviar medios específicos para auxiliar a los niños de esta escuela ya que justamente no están ahí en la tarde: la escuela solo recibe alumnos 


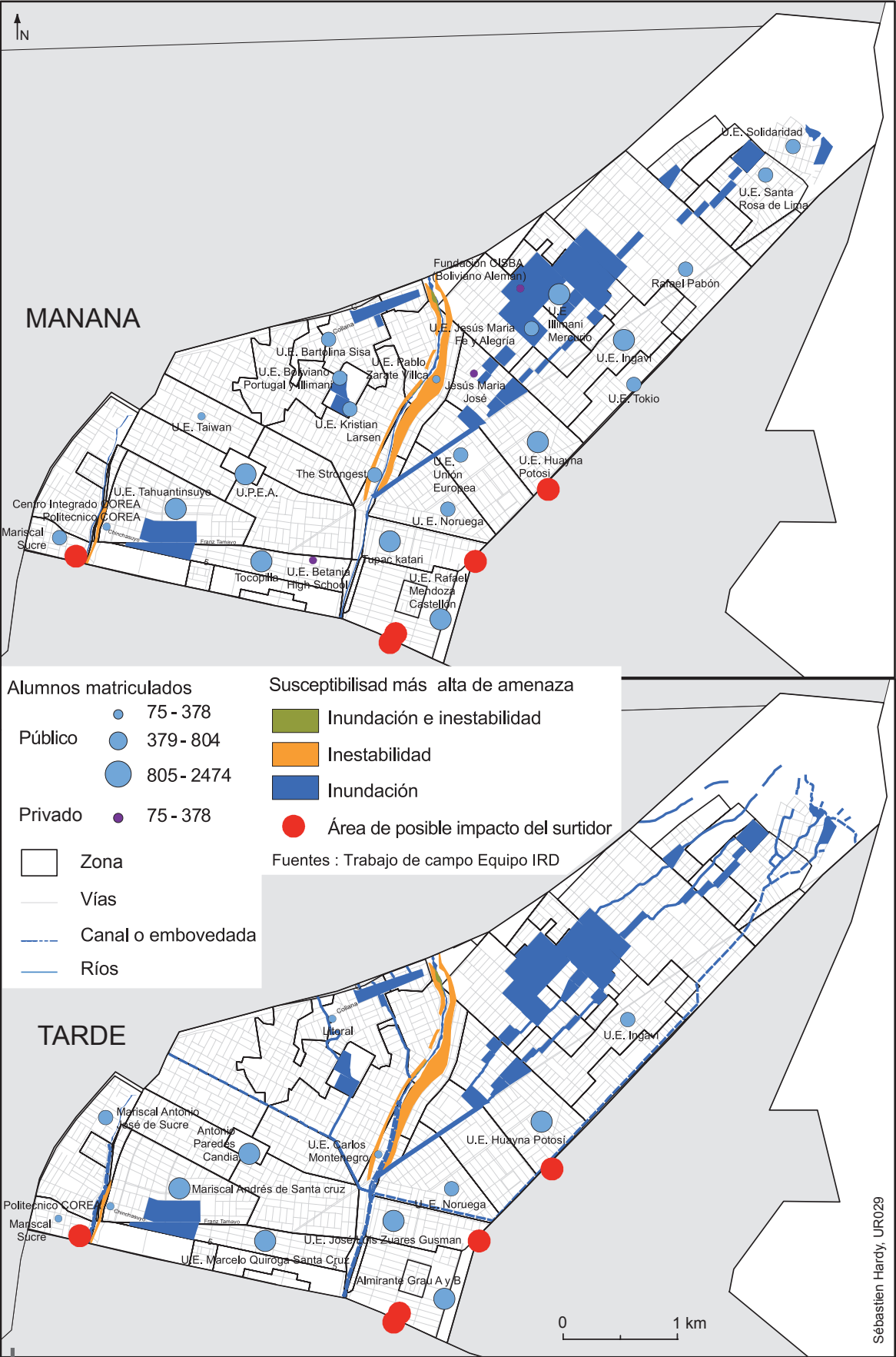

Figura 8 - La exposición a multiamenazas de los centros educativos del distrito 5: turno de la mañana - turno de la tarde 
en el turno de la mañana. Esta información les permite concentrarse en otros aspectos de las operaciones de auxilios que hay que llevar a cabo en la zona para reducir las consecuencias para las poblaciones. De este modo hacen más eficaces las operaciones de auxilio.

\section{CONCLUSIÓN}

Un proyecto de investigación puede, llevando a cabo una reflexión sobre los conceptos y métodos, desembocar en una aplicabilidad de los conocimientos que genera productos útiles para sus posibles beneficiarios.

En el caso de la temática de los riesgos, los proyectos Dipecho son particularmente propicios a este tipo de trabajo de investigación. Proporcionan un marco para crear nuevos conocimientos, permitiendo reflexionar sobre los conceptos y en sus diferentes dimensiones. En los distritos 5 y 6 de El Alto, se hizo una reflexión sobre las temporalidades de los riesgos. Poner en evidencia la temporalidad de ciertos elementos del funcionamiento urbano, principalmente de las poblaciones, revela las variabilidades espaciales de los elementos en función de las variabilidades temporales. La asimilación de este diagnóstico por los gestores de crisis, traducida por una herramienta cartográfica, hace que la gestión de crisis sea más eficaz. Esta debería permitir anticipar estas variabilidades y aprehender mejor la complejidad limitando la incertidumbre y por lo tanto, el daño posible. Por ejemplo, en El Alto los gestores han podido llevar a cabo campañas de sensibilización a los riesgos en los establecimientos escolares identificados por su susceptibilidad de ser dañados por exposición a las amenazas, integrando justamente la variabilidad espacio temporal por sus propios servicios de auxilio. Aunque por el momento la aplicación se muestra imposible, entre otros porque necesita de negociaciones delicadas con las asociaciones de comerciantes, estos han comprendido el interés de asegurar ciertas vías de acceso en la zona de la Feria 16 de Julio para limitar los daños durante la ocurrencia de un evento.

Esta reflexión se hizo también porque los conocimientos científicos deben ser útiles y aplicables para los beneficiarios de los proyectos. Un marco de investigación aplicada ofrece entonces una libertad de experimentación metodológica para responder a la presión de una mejor preparación a los daños. En los distritos 5 y 6 de la ciudad de El Alto, los conocimientos sobre los riesgos eran empíricos antes del comienzo del proyecto. Creando conocimientos —identificación de las amenazas y de los elementos expuestos, análisis de las condiciones de la vulnerabilidad entre las cuales la temporalidad-, el proyecto de investigación ha logrado ya el objetivo de proporcionar conocimientos útiles a los beneficiarios para aprehender las catástrofes. Por esto, ha permitido comprender cómo ocurren las situaciones de urgencia y de crisis, y proponer pistas de comprensión sobre la ocurrencia de las crisis, así como pistas de reflexión para prepararse ante ellas y reducirlas. 


\section{Referencias citadas}

ARBONA, J. \& KOHL, B., 2004 - City profile. La Paz-El Alto. Cities, vol. 21, n. ${ }^{\circ}$ 3: 255265.

ARGOLLO, J., 2008 - Estudio de identificación de zonas de riesgos en los distritos 5 y 6 de la ciudad de El Alto: análisis geológico-geomorfológico, 15 pp.; La Paz: IGEMA-IRD.

BABY, V., 1995 - El Alto de La Paz. Un bidonville d'altitude au cœur de l'Amérique latine, 142 pp. Mémoire de DEA, Université Paris X, Nanterre.

BCEOM, BRGM, PCA, 1977 - Plan de desarrollo urbano-ciudad de La Paz; La Paz: HAM, 41 tomes.

BLAIKIE, P., CANNON, T. \& WISNER, B., 1994 - At Risk. Natural Peoples's Vulnerability, and Disasters, 280 pp.; Londres: Routledge.

DAUPHINÉ, A., 2001 - Risques et catastrophes. Observer, spatialiser, comprendre, gérer, 288 pp.; París: Éditions Armand Colin.

DAUPHINÉ, A. \& PROVITOLO, D., 2007 - La résilience : un concept pour la gestion des risques. Annales de géographie, $\mathbf{n} .^{\circ}$ 654: 115-125.

DAVIS, M., 2006a - Génocides tropicaux. Catastrophes naturelles et famines coloniales. Aux origines du sous-développement, 479 pp.; París: Éditions La Découverte.

DAVIS, M., 2006b - Le pire des mondes possibles. De l'explosion urbaine au bidonville global, 250 pp.; París: Éditions La Découverte.

D'ERCOLE, R. \& METZGER, P., 2004 - La vulnerabilidad del Distrito Metropolitano de Quito, 496 pp.; Quito: MDMQ/IRD.

D'ERCOLE, R. \& METZGER, P., 2009 - Enjeux territoriaux et vulnérabilité : une approche opérationnelle. In: Risques et environnement : recherches interdisciplinaires sur la vulnérabilité des sociétés (A. Peltier \& S. Becerra, eds.): 391-402; París: Éditions L'Harmattan.

D'ERCOLE, R., GLUSKI, P., HARDY, S. \& SIERRA, A., 2009 - Vulnérabilités urbaines dans les pays du Sud. Présentation du dossier. Cybergeo: European Journal of Geography, [En ligne] Dossiers, Vulnérabilités urbaines au sud, URL: http://www.cybergeo.eu/ index22151.html

DUBOIS-MAURY, J. \& CHALINE, C., 2002 - Les risques urbains, 208 pp.; París: Éditions Armand Colin.

DURÁN, J., ARIAS, K. \& RODRÍGUEZ, M., 2007 - Aunque en la punta del cerro. Vivienda y desarrollo de la Ciudad de El Alto; La Paz: PIEB.

FRANQUEVILLE, A., 2000 - Du Cameroun à la Bolivie. Retours sur un itinéraire, 321 pp.; París: Karthala.

GARCÍA LINERA, Á., 2006 - Comunidades vecinales y lógica empresarial en la gestión del agua en la ciudad de El Alto. In: No somos juguete de nadie... Análisis de la relación de movimientos sociales, recursos naturales, Estado y descentralización (S. Orozco Ramírez, Á. García Linera \& P. Stefanoni, eds.): 247-322; La Paz: Plural Editores.

GARFIAS, S. \& MAZUREK, H., 2005 - El Alto desde una perspectiva poblacional, 130 pp.; La Paz: CODEPO-IRD.

GMLP, 2005 - Dossier estadístico del municipio de La Paz 2000-2005, 592 pp.; La Paz: GMLP.

GMLP, 2007 - Catálogo de instrumentos en gestión municipal para la reducción de riesgos y preparación ante emergencias, 74 pp.; La Paz: PNUD. 
HARDY, S. \& MUSSET, A., 2008 - Zentralamerika: Naturbedingte Risiken und soziale Verwundbarkeit. In: Zentralamerika Heute (S. Kurtenbach, W. Mackenbach, G. Maihold \& W. Wünderich, eds.): 85-102; Frankfurt am Main: Vervuert Verlag.

HARDY, S., 2008 - Estudio de identificación de zonas de riesgos en los distritos 5 y 6 de la ciudad de El Alto, 66 pp.; La Paz: ECHO-Coopi [En línea] URL: http://www. documentation.ird.fr/fdi/notice.php?ninv=fdi:010045023

HARDY, S., 2009a - Explorer la construction de la résilience. Expériences de recherche à La Paz. In: Risques et environnement : recherches interdisciplinaires sur la vulnérabilité des sociétés (A. Peltier \& S. Becerra, eds.): 469-482; París: Éditions L'Harmattan.

HARDY, S., 2009b - La vulnérabilité de l'approvisionnement en eau dans I'agglomération pacénienne. Le cas du sous-système El Alto. Cybergéo: European Journal of Geography [En ligne], Dossiers, Vulnérabilités urbaines au sud, document 457, mis en ligne le 20 mai 2009. URL : http://www.cybergeo.eu/index22270.html

HARDY, S., por publicarse - Modificar la definición del riesgo para que la investigación sea verdaderamente aplicable. Demostración a partir del caso de Managua. TRACE.

INE, 2001 - Censo 2001. Población por organizaciones comunitarias y localidades; La Paz: INE.

INE, 2008 - La Paz: poblacion total proyectada, 2005-2007, URL: http://www.ine.gov.bo.

LAGADEC, P., 2002 - Crisis Management in France: Trends, Shifts and Perspectives. Journal of contingencies and crisis management, vol. 10, $\mathbf{n} .^{\circ} \mathbf{4}$ : 159-172.

MAYORGA, F., 1997 (ed.) - ¿Hegemonías? Democracia representativa y liderazgos locales, 380 pp.; La Paz: PIEB.

MOLLERICONA, J., 2008 - Estudio de identificación de zonas de riesgos en los distritos 5 y 6 de la ciudad de El Alto: análisis de la percepción de los riesgos, 50 pp.: La Paz: UPIEB-IRD.

MONTES DE OCA, I., 2005 - Enciclopedia geográfica de Bolivia, 871 pp.; La Paz, Editores Atenea.

OBERMAIER, S., 1999 (ed.) - Plan regulador de la ciudad de El Alto, 81 pp.; El Alto: BID.

OBERMAIER, S., 2000 (ed.) - Plan de ordenamiento urbano de la ciudad de El Alto, 77 pp.; El Alto: BID.

OLMOS, C., 2008 - Estudio de identificación de zonas de riesgos en los distritos 5 y 6 de la ciudad de El Alto: análisis de hidrología, 77 pp.; La Paz: IHH-IRD.

PELLING, M., 2003 - The Vulnerability of Cities-natural Disasters and Social Resilience, 256 pp.; Londres: James/Earthscan.

PIGEON, P., 2005 - Géographie critique des risques, 217 pp.; París: Economica/Anthropos. PIGEON, P., 2007 - L'environnement au défi de l'urbanisation, 189 pp.; Rennes: PUR.

POUPEAU, F., 2007 - Movilizaciones políticas y gestión de la penuria de agua en los distritos populares de El Alto, Bolivia. In: Después de las guerras del agua, movilizaciones políticas: 209-235; La Paz: Plural.

POUPEAU, F., 2008a - Carnets boliviens 1999-2007. Un goût de poussière, 216 pp.; Montreuil: Aux lieux d'être.

POUPEAU, F., 2008b - Les ambivalences de la participation communautaire. Le cas du service de distribution des eaux à El Alto, Bolivie. Autrepart, n. ${ }^{\circ}$ 47: 245-253.

ROBERT, J., 2007 - Vulnérabilité de la population exposée aux lahars du volcan Cotopaxi dans le Valle de Los Chillos, Quito (Équateur). Caractérisation, quantification et cartographie de la population, 68 pp. Mémoire de Master 2 Systèmes territoriaux, développement durable et aide à la décision, Chambéry, Université de Savoie. 
SANDOVAL, G. \& AYLLON, V., 1992 - La memoria de las ciudades (bibliografía urbana de Bolivia 1952-1991), 499 pp.; La Paz: CEP-ILDIS.

SANDOVAL, G. \& SOSTRES, F., 1989 - La ciudad prometida, La Paz: SYSTEMA-ILDIS. 